\section{Chemical Structure and Serological Activity of Synthetic and Natural Cardiolipin}

CARdiolipin was first isolated by Pangborn ${ }^{1}$ from ox-heart and this compound proved to be the essential lipid of the antigen in the serodiagnosis of syphilis. Based on hydrolysis investigations, Macfarlane et al. ${ }^{2,3}$ and Faure and Morelec-Coulon ${ }^{4,5}$ arrived at the conclusion that cardiolipin has a diphosphatidyl glycerol (I) structure. Recently, LeCocq and Ballou ${ }^{6}$ confirmed this view,

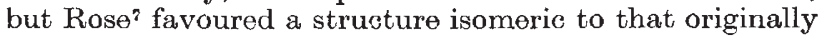
proposed by Pangborn ${ }^{8}$. Conclusive evidence about the structure of cardiolipin has now been obtained by means of chemical synthesis.

We have succeeded in the preparation of diphosphatidyl glycerol (I) by a condensation reaction between silver $\gamma$-stearoyl- $\beta$-oleoyl-L- $\alpha$-glycerobenzyl phosphate and $\alpha, \gamma$ di-iodo glyeero- $\beta$-t. butylether followed by removal of the protecting benzyl and $t$.butylether functions ${ }^{9}$. Comparison of ox-heart cardiolipin with the synthesized compound revealed no differences in chromatographical behaviour, infra-red absorption, melting point and optical rotation (natural cardiolipin $[\alpha]_{D}^{20}+5 \cdot 5^{\circ}$; synthesized diphosphatidyl glycerol $[\alpha]_{D}^{20}+5 \cdot 8^{\circ}$, both in chloroform $c$ (ref. 10)). Mild alkaline hydrolysis of the natural cardiolipin and the synthetic material yielded water-soluble phosphate esters indistinguishable by paper electrophoresis and chromatography. Final proof for the identity of natural cardiolipin and synthetic diphosphatidyl glycerol was obtained by enzymatic hydrolysis with the phospholipases $A(E C$ 3.1.1.4) and $C(E C$ 3.1.4.3). Short. timo incubation of both phospholipids with Crotalus adamanteus venom (in the presence of stearylphosphoryl choline which reduces the negative charge of the lipid micelles) resulted in the formation of two lyso-derivatives lacking one and two $\beta$-fatty acids respectively. After prolonged incubation both phospholipids were quantita tively converted into one slow-moving lysophospholipid containing half the amount of fatty acids originally present ${ }^{10}$.

In accordance with the findings of Ottolenghi ${ }^{11}$ the action of phospholipase $C$ appeared to be catalysed by zinc ions. A short incubation of both substrates with the enzyme of Bacillus cereus yielded a 1,2-diglyceride and a phospholipid which was found to be chromatographically identical with chemically synthesized phosphatidyl glycerophosphat ${ }^{12}$. Prolonged action of this enzyme converted both the synthetic and isolated cardiolipin completely into 1,3-diphosphoglycerol (not contaminated with the 1,2-isomer) and 1,2-diglyceride. These findings unequivocally demonstrate that cardiolipin from ox-heart is identical to 1,3-diphosphatidyl glycerol. A great number of synthetic acidic phosphatides have been investigated as possible substitutes for cardiolipin in the sero. diagnostic tests for syphilis ${ }^{13,14}$; none of these synthetic samples revealed an activity which equalled that of oxheart cardiolipin. The serological activity of our synthetic product was compared with that of ox-heart cardiolipin by Dr. J. H. de Bruijn of the National Institute of Public

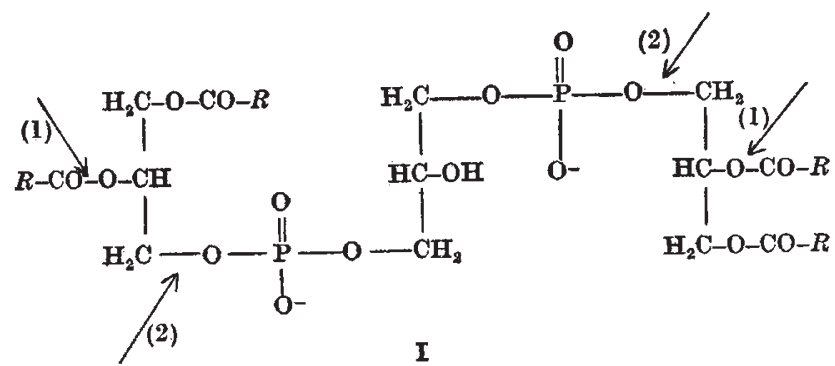
Fig. 1. Structural formula of diphosphatidyl glycerol (cardiolipin).
Arrows (1) and (2) indicate the sites of attack of phospholipase $A$ and phospholipase $C$ respectively
Health (Utrecht). At present more than one hundred sera have been investigated. In the Kolmer complement fixation test the reactivity of the synthesized diphosphatidyl glycerol appeared to be indistinguishable from those of ox-heart cardiolipin. In the VDRL slide test the synthesized compound gave even more clear-cut positive results without altering the specificity of the reaction. It should be noted that ox-heart cardiolipin consists of about 80 per cent of linoleic acid in contrast to our synthetic product, which contains equimolar amounts of stearic and oleic acid. The results obtained in the serological assays indicate that the degree of unsaturation of the fatty acids apparently is not of primary importance for the activity of cardiolipin in this reaction. With regard to the relation between structure and activity of acidic phospholipids in the serological assays for diagnosis of syphilis, it will be of interest to evaluate the significance of the zeta-potential of the micelles of the lipid antigen.

$$
\begin{aligned}
& \text { G. H. DE HaAs } \\
& \text { L. L. M. VAN DeENeN }
\end{aligned}
$$

Department of Biochemistry,

Laboratory of Organic Chemistry, State University,

Utrecht, The Netherlands.

1 Pangborn, M. C., J. Biol. Chem., 143, 247 (1942).

2 Macfarlane, M. G., and Gray, G. M., Biochem, $J .67,25 \mathrm{P}(1957)$

${ }^{3}$ Macfarlane, M. G., and Wheeldon, L. W., Nature, 183, 1808 (1959).

${ }^{4}$ Faure, M., and Morelec-Coulon, M. J., Ann. Inst. Pasteur, 91, 537 (1956).

5 Faure, M., and Morelec-Coulon, M. J., Bull. Soc. Chim. Biol., 42, 867 (1960).

${ }^{8}$ LeCoca, J., and Ballou, C. E., Biochemistry, 3, 976 (1964).

7 Rose, H. G., Biochim. Biophys. Acta, 84, 109 (1964).

8 Pangborn, M. C., J. Biol. Chem., 168, 351 (1947).

- de Haas, G. H., and van Deenen, L. L. M., Rec. Trav. Chim., 84, 436 (1965). ${ }^{10}$ van Deenen, L. L. M., and de Haas, G. H., Biochim. Biophys. Acta, 70, 538 (1963).

11 Ottolenghi, A. C., Fed. Proc., 23 (1964).

${ }^{2}$ Bonsen, P. P. M., de Haas, G. H., and van Deenen, L. L. M. (submitted for publication).

${ }^{13}$ Allen, R. H., and Tonks, D. B., Bull. World Health Org., 19, 547 (1958).

${ }^{14}$ Foit, E., and Schindler, M., Der Hautarzt, 7, 210 (1956).

\section{'Isoallergens' from Rye Grass Pollen}

SEveral methods for the purification of allergens from pollens and from other vegetable and animal sources have been reported ${ }^{1-9}$, but recently, by utilizing highly selective chromatographic procedures, it has been possible to obtain allergens of much higher purity than hitherto described ${ }^{10,11}$. At least two groups of allergens have been found in rye pollen, a major group, active in the majority of sensitive patients, and a minor group, active in only a small proportion. It seems likely that such groups also exist in other grass and ragweed pollens causing hay-fever.

The method of isolating rye pollen allergens, described in detail elsewhere ${ }^{10}$, was briefly as follows. Etherdefatted rye grass pollen was extracted with $0.001 \mathrm{M}$ $\mathrm{NH}_{4} \mathrm{HCO}_{3}$ at $p \mathrm{H} 8$ and the clarified extract was dialysed against the same solvent. The allergenic non-dialysable material was then concentrated by freeze-drying (yield: $6 \mathrm{~g} / 100 \mathrm{~g}$ defatted pollen). This process has repeatedly been shown to yield virtually all the allergenic substances ${ }^{1,3,10,12}$, assayed by quantitative skin testing on allergic volunteers ${ }^{13}$. Allergenic components were then isolated by fractionation of dialysed extracts successively on columns of DEAE-cellulose and 'Sephadex G75'. Homogeneity of the fractions was assessed by starch-gel electrophoresis (Fig. 1), ultracentrifugation, behaviour on 'Sephadex $G 75$ ', N-terminal amino-acid analysis and immuno-diffusion against rabbit anti-rye pollen sera. Their skin activity, relative to whole-pollen extract, was shown to increase at each stage of purification of the major allergenic fractions.

Two allergens, $\alpha$ and $\beta$, were each homogeneous according to the foregoing criteria and by gel-diffusion assay appeared to be immunologically identical (Fig. 2 and ref. 